\title{
Hippocampal-Prefrontal BDNF and Memory for Fear Extinction
}

\author{
Luis E Rosas-Vidal', Fabricio H Do-Monte', Francisco Sotres-Bayon ${ }^{1,2}$ and Gregory J Quirk*,' \\ 'Departments of Psychiatry and Anatomy and Neurobiology, University of Puerto Rico, University of Puerto Rico School of Medicine, San Juan, \\ Puerto Rico
}

\begin{abstract}
Infusing brain-derived neurotrophic factor (BDNF) into the infralimbic (IL) prefrontal cortex is capable of inducing extinction. Little is known, however, about the circuits mediating BDNF effects on extinction or the extent to which extinction requires BDNF in IL. Using local pharmacological infusion of BDNF protein, or an antibody against BDNF, we found that BDNF in the IL, but not prelimbic (PL) prefrontal cortex, is both necessary and sufficient for fear extinction. Furthermore, we report that BDNF in IL can induce extinction of older fear memories ( 14 days) as well as recent fear memories (I day). Using immunocytochemistry, we show that BDNF is increased in the ventral hippocampus (VHPC), but not IL or PL, following extinction training. Finally, we observed that infusing BDNF into the $V H P C$ increased the firing rate of $\mathrm{IL}$, but not $\mathrm{PL}$ neurons in fear conditioned rats. These findings indicate that an extinction-induced increase in BDNF within the $\mathrm{VHPC}$ enhances excitability in IL targets, thereby supporting extinction memories.
\end{abstract}

Neuropsychopharmacology (2014) 39, 2 I6I-2I69; doi:I0.1038/npp.2014.64; published online 26 March 2014

\section{INTRODUCTION}

There is growing interest in the mechanisms of BDNF effects on plasticity underlying fear extinction learning (Andero and Ressler, 2012; Cowansage et al, 2010). The infralimbic (IL) prefrontal cortex is essential for fear extinction learning (Herry et al, 2010; Pape and Pare, 2010; Quirk and Mueller, 2008). Humans and rodents with a polymorphism that reduces BDNF release (Chen et al, 2006) are impaired in extinction learning (Soliman et al, 2010), and mice with this polymorphism show impaired synaptic transmission and plasticity in IL (Pattwell et al, 2012). Furthermore, extinction training increases BDNF mRNA in IL (Bredy et al, 2007).

Consistent with these rodent findings, we recently reported that infusing BDNF into IL facilitates extinction, even in the absence of training, suggesting that BDNF in IL can be sufficient for extinction (Peters et al, 2010). It is not known, however, if BDNF in the neighboring prelimbic (PL) prefrontal cortex may be involved, or the extent to which these BDNF effects depend on the age of the fear memory. Also unknown is the extent to which IL BDNF is necessary for extinction, or whether IL BDNF is derived from inputs from the basolateral nucleus of the amygdala (BLA) or the

\footnotetext{
*Correspondence: Dr G] Quirk, Departments of Psychiatry and Anatomy and Neurobiology, University of Puerto Rico, School of Medicine, PO Box 365067, San Juan 00936-5067, Puerto Rico, Tel: +787 999 3058, Fax: +787 999 3057, E-mail: gregoryjquirk@gmail.com ${ }^{2}$ Current address: Instituto de Fisiología Celular-Neuorciencias, Univ Nacional Autónoma de México, México DF 04510, México

Received 27 November 20 13; revised 20 February 2014; accepted 10

March 2014; accepted article preview online I4 March 2014
}

ventral hippocampus (vHPC; Hoover and Vertes, 2007). Knocking down BDNF or its signaling in either of these structures impairs extinction learning (Chhatwal et al, 2006; Heldt et al, 2007, 2014).

To address these issues, we compared the effects of IL $v s$ PL infusions of BDNF, and varied the age of the fear memory. We also attempted to block extinction by infusing an antibody against BDNF. Finally, we used immunocytochemistry and unit recording to determine the extent to which BDNF expression is modified within the prefrontalhippocampal-amygdala circuit. Our findings support the hypothesis that neuronal BDNF in the hippocampal-IL circuit facilitates extinction of older, as well as recent, fear memories.

\section{MATERIALS AND METHODS}

\section{Subjects}

A total of 156 male Sprague-Dawley rats (Harlan Laboratories, Indianapolis, IN) were housed and handled as described previously (Quirk et al, 2000). Briefly, rats were restricted to $18 \mathrm{~g}$ per day of standard laboratory rat chow, and trained to press a bar for food on a variable interval schedule of reinforcement (VI $60 \mathrm{~s}$ ). Pressing for food provides a baseline of activity against which freezing can be reliably measured (Quirk et al, 2000). All procedures were approved by the University of Puerto Rico School of Medicine Animal Care and Use Committee, in compliance with the National Institutes of Health guidelines for the care of laboratory animals. 


\section{Surgery}

Following bar-press training, rats were anesthetized under isoflurane anesthesia and 26-gauge guide bilateral cannulas (Plastics One, Roanoke, VA) were bilaterally implanted in the IL cortex (coordinates: +2.8 AP; $\pm 3.1 \mathrm{ML} ;-3.8 \mathrm{DV}$; angled at $30^{\circ}$ ), the PL cortex (coordinates: $+2.8 \mathrm{AP} ; \pm 0.6$ $\mathrm{ML} ;-2.6 \mathrm{DV})$, or the vHPC $(-6.0 \mathrm{~mm} \mathrm{AP}, \pm 5.0 \mathrm{~mm} \mathrm{ML}$, lateral, $-6.0 \mathrm{~mm} \mathrm{DV}$; Paxinos and Watson, 1998). For IL cannula implantation, an angled approach was used to avoid backflow to PL (Sierra-Mercado et al, 2011). The cannulas were fixed to the skull using acrylic cement and three skull screws. Stainless-steel obturators (33 gauge) were inserted into the guide cannulas to avoid obstruction until infusions were made. For unit recording experiments, a cannula was implanted unilaterally in the vHPC $(-6.0 \mathrm{~mm}$ $\mathrm{AP}, \pm 5.0 \mathrm{~mm} \mathrm{ML}$, lateral, $-6.0 \mathrm{~mm} \mathrm{DV}$ ), ipsilateral to the recording electrode, which consisted of either a movable bundle of 16 microwires ( $25 \mu \mathrm{m}$ in diameter; Stablohm 675; California Fine Wire, Grover Beach, CA) or a movable $3 \times 3$ electrode array (Neuro Biological Laboratories, Denison, TX). Before electrode bundles were implanted, impedance was reduced to $250-350 \mathrm{k} \Omega$ by passing a $1 \mathrm{~mA}$ cathodal current in a gold solution (arrays were not gold plated). The electrode bundle/array was implanted in IL $(+3.0 \mathrm{~mm} \mathrm{AP}$, $+0.5-0.8 \mathrm{~mm} \mathrm{ML},-5.0 \mathrm{~mm} \mathrm{DV})$ or $\mathrm{PL}(+3.0 \mathrm{~mm} \mathrm{AP}$, $+0.5-0.8 \mathrm{~mm} \mathrm{ML},-4.0 \mathrm{~mm} \mathrm{DV})$. Following surgery, triple antibiotic was applied to the wound and ketoprofen $(5 \mathrm{mg} /$ $\mathrm{kg}$ ) was injected intramuscularly as an analgesic and antiinflammatory agent. Rats were allowed to recover for 5-7 days before initiating experiments.

\section{Behavior}

Auditory fear conditioning and extinction were performed in the same operant chambers (Coulbourn Instruments, Allentown, PA) located in sound-attenuating cubicles (Med Associates, Burlington, VT) throughout all phases of the experiment. The floor of the chambers consisted of stainless-steel bars that delivered a scrambled electric foot shock. Between experiments, shock grids and floor trays were cleaned with soap and water, and the chamber walls were cleaned with wet paper towels. On day 1 , rats received five habituation tones $(30 \mathrm{~s}, 4 \mathrm{kHz}, 75 \mathrm{~dB} ; 3 \mathrm{~min}$ intertrial interval), immediately followed by seven conditioning tones that coterminated with foot shocks $(0.5 \mathrm{~s}, 0.5 \mathrm{~mA})$. On day 2 (recent memory BDNF experiments) or day 14 (older memory BDNF experiment), rats were infused with BDNF and returned to their home cages. For the anti-BDNF experiments, rats were infused with anti-BDNF and placed in the conditioning chambers for extinction training, consisting of 20 tone presentations in the absence of footshock. The following day (day 3 or day 15), rats were returned to the chambers and presented with either two tones (BDNF experiments) or eight tones (anti-BDNF experiments) to test for extinction retrieval. For the BDNF immunocytochemistry experiments, rats were killed 1 or $2 \mathrm{~h}$ following extinction tones on day 2. For the single-unit recording experiments, conditioned rats were returned to the training chamber and presented with two tones. BDNF was then infused into VHPC and two additional tones were presented $30 \mathrm{~min}$ following the infusion. Food pellets were available in the conditioning chambers on a VI $60 \mathrm{~s}$ schedule throughout all phases of the experiment.

\section{Histology}

After behavioral experiments, rats were deeply anesthetized with sodium pentobarbital ( $450 \mathrm{mg} / \mathrm{kg}$ intraperitoneally) and transcardially perfused with $0.9 \%$ saline followed by $10 \%$ buffered formaldehyde. In addition, to assist with localization of electrode placement, a microlesion was made by passing an anodal current $(20 \mathrm{~mA}$ for $20 \mathrm{~s})$ through the electrode. Brains were removed and stored in a $30 \%$ sucrose $/ 10 \%$ formaldehyde solution for at least $48 \mathrm{~h}$ before sectioning. Six percent of ferrocyanide was included in the solution to stain the iron deposited following the electrode microlesion. Coronal sections were cut $40 \mu \mathrm{m}$ thick, mounted on slides, and stained for Nissl bodies.

\section{Drug Infusions}

For drug infusions, obturators were removed and injectors were placed into the guide cannulas. Injector tips extended $1 \mathrm{~mm}$ beyond the guide cannula. One day before testing, rats were habituated for handling and injectors were passed through the cannula (without infusion). Drugs were dissolved in physiological saline and infused at a rate of $0.25 \mu \mathrm{l} / \mathrm{min}$. Following infusion, injectors were left in place for at least $1 \mathrm{~min}$ to allow for diffusion of the drug. Recombinant human BDNF (R\&D Systems, Minneapolis, MN USA) was administered at a dose of $0.75 \mu \mathrm{g} / 0.5 \mu \mathrm{l}$ per side (Peters et al, 2010). Animals were returned to their home cage or to the conditioning chamber after infusion. Sheep anti-BDNF antibody (EMD Millipore, Billerica, MA) was administered at a dose of $0.5 \mu \mathrm{g} / 0.5 \mu \mathrm{l}$ per side (Peters et al, 2010). Infusions were performed $10-30 \mathrm{~min}$ before extinction training. Animals were infused with drugs only once.

\section{Immunocytochemistry}

Rats were deeply anesthetized with sodium pentobarbital ( $450 \mathrm{mg} / \mathrm{kg}$ intraperitoneally) 1 or $2 \mathrm{~h}$ after concluding the corresponding behavioral session. They were perfused transcardially with $100 \mathrm{ml}$ of $0.9 \%$ saline followed by $500 \mathrm{ml}$ of $4 \%$ paraformaldehyde in $0.1 \mathrm{M}$ phosphate buffer at $\mathrm{pH}$ 7.4. Brains were removed from the skull and fixed overnight in the same fixative solution. The next day, brains were transferred to a solution of $30 \%$ sucrose in $0.1 \mathrm{M}$ phosphate buffer at $4{ }^{\circ} \mathrm{C}$ during $48 \mathrm{~h}$ for cryoprotection. The brains were frozen and a series of coronal sections $(40 \mu \mathrm{m})$ were cut on a cryostat (CM 1850; Leica) and collected at different levels of the medial prefrontal cortex, vHPC, and amygdala.

Alternate sections were initially blocked in a solution of $2 \%$ normal goat serum (Vector Laboratories, Burlingame, CA) plus $0.3 \%$ Triton X-100 (Sigma-Aldrich, St Louis, MO) in $0.12 \mathrm{M}$ potassium phosphate buffered saline for $1 \mathrm{~h}$ and then incubated overnight at room temperature with sheep anti-BDNF antibody (1:200; AB1513P; EMD Millipore) plus anti-NeuN $(1: 200$, conjugated with rabbit polyclonal Alexa Fluor 488; EMD Millipore). This anti-BDNF antibody was successfully used for immunocytochemistry in previous studies (Gunjigake et al, 2006; Ou et al, 2010). The next day, slices were incubated with anti-sheep fluorescent secondary 
antibody (1:200; Alexa Fluor 594; Invitrogen, Carslbad, CA) for $2 \mathrm{~h}$, mounted on gelatin-coated slides, dehydrated and then coverslipped with antifading mounting medium (Vectashield; Vector Laboratories, Burlingame, CA). Our immunocytochemical procedure for BDNF and NeuN was adapted from a previous study (Ou et al, 2010).

Images were digitized with a microscope (Model BX51; Olympus, Tokyo, Japan) coupled to a fluorescent lamp (X-Cite Series 120Q; Lumen Dynamics, Ontario, Canada) at $\times 20$ magnification using a digital camera (Model DP72; Olympus, Tokyo, Japan). Images were generated for PL, IL, vHPC, basal nucleus of the amygdala (BA), lateral portion of the central nucleus of the amygdala (CeL), and medial portion of the central nucleus of the amygdala (CeM), using the appropriate filter sets for Alexa 488 and Alexa 594. Images were processed using commercial software (Metamorph version 6.1; Molecular Devices, Sunnyvale, CA) by digitally removing background luminescence and automatically determining the threshold. BDNF colocalization with NeuN was quantified by measuring the percentage of overlap between the NeuN and BDNF labeling as described previously (Do-Monte et al, 2013). The percentage of overlapping area was averaged for the two hemispheres at two to three distinct rostrocaudal levels of each region.

\section{Data Collection and Analysis}

Behavior was recorded with digital video cameras (Micro Video Products, Bobcaygeon, ON, Canada). In behavioral experiments, freezing was quantified from digitized video images using commercially available software (Freezescan, Clever Systems, Reston, VA). The amount of time spent freezing to the tone was expressed as a percentage of the tone presentation. Trials were averaged in blocks of two, and subjected to Student's two-tailed $t$-tests or repeatedmeasures analysis of variance (ANOVA), followed by Tukey's post hoc comparisons as appropriate (STATISTICA; Statsoft, Tulsa, OK).

\section{Single-Unit Recordings}

Extracellular waveforms exceeding a voltage threshold were amplified (gain $100 \times$ ), digitized at $40 \mathrm{kHz}$ using a Multichannel Acquisition Processor System (Plexon, Dallas, TX), and stored onto disk for further off-line analysis. Waveforms were recorded during pretone, tone, and posttone periods, each lasting $30 \mathrm{~s}$. Single units were isolated using principal component analysis and template matching (Offline Sorter; Plexon). Both automated and manual correction processing techniques were applied to sort spikes (see Burgos-Robles et al, 2013; Sotres-Bayon et al, 2012). Automated processing was carried out using a valleyseeking scan algorithm (Offline Sorter; Plexon), one channel at a time, and then evaluated using sort quality metrics. For manual verification of automated clustering techniques, a cluster was considered for analysis if it was discretely separated from other clusters in principal component space. In addition, spikes with interspike intervals $<1 \mathrm{~ms}$ were excluded. Only stable clusters of single units were considered for analysis. Timestamps of neural spiking were analyzed with NeuroExplorer (NEX Technologies, Littleton, $\mathrm{MA})$. Neuronal activity was recorded before and $30 \mathrm{~min}$ after infusion of BDNF into vHPC. To assess whether BDNF altered spontaneous firing rates, ten 3-s bins were compared before and after BDNF infusion, using paired Student's $t$-tests. Units with initial firing rates $>12 \mathrm{~Hz}$ (presumed interneurons) were excluded from analysis (IL: $n=1$; PL: $n=3)$.

\section{RESULTS}

\section{BDNF Infused into IL, but not PL, is Sufficient for Extinction}

In our first experiment, BDNF was infused into either IL or $\mathrm{PL}$ of rats previously given auditory fear conditioning. On day 1, rats were fear conditioned and divided into two groups matched for freezing levels. The next day (day 2), rats were infused with either BDNF or saline in IL through angled cannulas, returned to their home cages, and tested the following day (day 3) drug free. As illustrated in Figure 1a, there was a significant reduction in freezing in rats infused with BDNF (Sal: 68.6\%; BDNF: $34.6 \% ; t_{(19)}=2.66 ; P=0.015$ ), replicating our previous findings with vertical cannulas (Peters et al, 2010). A similar extinction-inducing effect of BDNF was observed in rats conditioned 13 days before BDNF infusion (see Figure $1 \mathrm{~b}$ ). When tested the following day (day 15), rats infused with BDNF showed a significant reduction in freezing compared with saline controls (Sal: 88.0\%; BDNF: $\left.61.4 \% ; t_{(15)}=3.00 ; P=0.009\right)$, suggesting that BDNF in IL is sufficient for extinction of older fear memories. In both experiments, bar pressing for food before the first tone was similar between groups (day 3-Sal: 12.5 presses per min, BDNF: 14.9 presses per min; day 15-Sal: 10.9 presses per min, BDNF: 13.2 presses per min; all $P$ 's $>0.63$ ), suggesting that reduction in tone fear by BDNF was not due to a decrease in contextual fear. In contrast to IL, infusion of BDNF into PL had no effect (see Figure 1c). One day following the PL infusion, rats receiving BDNF and saline showed equivalent levels of freezing (Sal: 73.2\%; BDNF: $\left.77.0 \% ; t_{(15)}=-0.040 ; P=0.969\right)$, suggesting that $\mathrm{PL}$ does not contribute to BDNF-induced fear extinction.

\section{BDNF in IL, but not PL, is Necessary for Extinction}

The above experiments confirm that administering BDNF in IL can induce extinction-like behavior, but they do not indicate whether endogenous BDNF in IL is necessary for extinction. We therefore infused a BDNF binding antibody (anti-BDNF) into IL to sequester endogenous BDNF and thus block its activity. This antibody (at the same dose) has been shown to impair BDNF-dependent signaling in the hippocampus (Alonso et al, 2002). Figure 2a shows that infusion of anti-BDNF into IL before extinction (day 2) did not alter freezing levels in the first block of extinction training (Sal: 65.2\%; anti-BDNF: 76.9\%; $t_{(19)}=-1.297$; $P=0.210)$. Anti-BDNF-infused rats extinguished more slowly than controls (main effect of group: $F_{(1,26)}=4.912$; $P=0.036$ ). Post hoc comparisons showed that freezing in the anti-BDNF group was significantly increased in block 9 $(P=0.048)$. The following day (day 3), freezing levels differed significantly between groups $\left(\mathrm{F}_{(1,26)}=5.231 ; P=0.036\right)$. Post hoc comparisons confirmed that freezing in the anti-BDNF group was increased in blocks $1(P=0.018)$ 
a

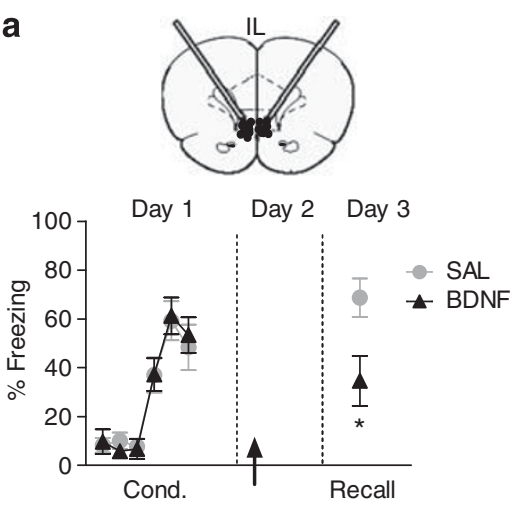

b
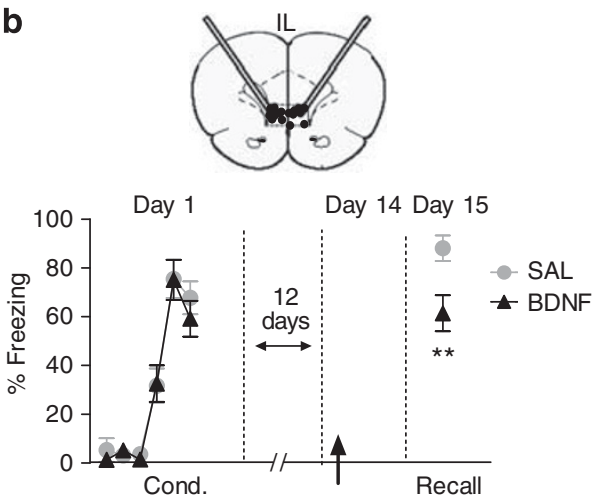

C
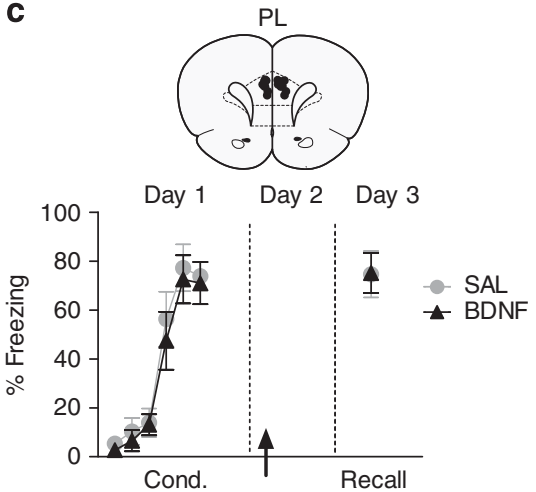

Figure I Brain-derived neurotrophic factor (BDNF) in infralimbic (IL), but not prelimbic (PL), prefrontal cortex induces extinction. (a, upper) Coronal drawings showing the angled cannulation approach and location of the injector tips in IL. (Lower) Infusion of BDNF in IL on day 2 (arrow) significantly decreased freezing on day 3 (Sal: $n=1$ I; BDNF: $n=10$ ). (b, upper) Coronal drawings showing the angled cannulation approach and location of the injector tips in IL. (Lower) Infusion of BDNF in IL 2 weeks after conditioning on day I4 (arrow) significantly decreased freezing on day I5 (Sal: $n=9$; BDNF: $n=8$ ). (c, upper) Coronal drawings showing the location of the injector tips in PL. (Lower) Infusion of BDNF in PL on day 2 (arrow) did not alter freezing levels on day 3 (Sal: $n=9 ;$ BDNF: $n=8$ ). Unpaired Student's t-test. Data are shown as mean \pm SEM in blocks of two trials. $* P<0.05, * * P<0.0$ l.

and $2(P<0.001)$. These results suggest that endogenous BDNF in IL is necessary for extinction.

In contrast to IL, infusion of anti-BDNF into PL did not alter fear expression or extinction learning (Figure 2b). Repeated-measures ANOVA revealed no effect of treatment $\left(\mathrm{F}_{(1,16)}<0.001 ; P=0.979\right)$ or interaction between treatment and trial block $\left(\mathrm{F}_{(9,144)}=1.195 ; P=0.302\right)$. On day 3 , rats tested drug free showed no group differences $\left(\mathrm{F}_{(1,16)}=\right.$ $0.017 ; P=0.896)$. Thus, as suggested by infusions of BDNF, PL does not appear to be a locus for BDNF-related extinction processes.

\section{Extinction Increases BDNF Expression in the vHPC and the Basal Amygdala}

There are multiple possible sources of extinction-related BDNF in IL, including BLA inputs, vHPC inputs (Hoover and Vertes, 2007), or IL itself. To address possible sources, we sought to determine which of these structures showed extinction-related increases in neuronal BDNF expression. Conditioned animals received either a complete set of 20 extinction trials (Extinction group), or only 2 extinction trials (No Extinction group; see Figure 3a). Both groups were in the test chamber for $71 \mathrm{~min}$ with access to food, and then returned to their home cages for either 1 or $2 \mathrm{~h}$, and then killed for immunocytochemistry. Neuronal levels of BDNF were indicated by the area of overlap between BDNF protein (in the cytoplasm) and NeuN, a marker of neuronal nuclei (Mullen et al, 1992; see Figure $3 \mathrm{~b}$ and $\mathrm{c}$ ).

As shown in Figure $3 \mathrm{~d}$ and e, Extinction and No Extinction groups did not significantly differ in neuronal BDNF levels in IL or PL, at either 1 or $2 \mathrm{~h}$ after extinction (all P's >0.459). In vHPC, however, the Extinction group showed significantly more neuronal BDNF than the No Extinction group at both timepoints (1 h-Extinction: 8.56\%; No Extinction: 4.14\%; $\mathrm{F}_{(1,14)}=5.00, \quad P=0.042 ; \quad 2 \mathrm{~h}-$ Extinction: 16.35\%; No Extinction: 9.19\%; $\mathrm{F}_{(1,6)}=8.53$, $P=0.027)$. In the amygdala, ANOVA showed a main effect of group $\left(\mathrm{F}_{(1,18)}=7.064 ; P=0.016\right)$ at $2 \mathrm{~h}$ but not $1 \mathrm{~h}$. Post hoc comparisons revealed an extinction-induced BDNF expression in BA $(P=0.038)$, but not in CeL or CeM. Thus, extinction-induced expression of BDNF in vHPC appears to precede that of BA, a target region of vHPC (Orsini et al, 2011).

\section{BDNF Infused into vHPC Decreases Fear and Increases the Activity of IL Neurons}

Extinction-induced BDNF in vHPC could influence extinction via IL, as suggested by Peters et al (2010), who showed that induction of extinction with hippocampal BDNF could be blocked by coinfusion of anti-BDNF into IL. Given that extinction training itself increases IL excitability (Chang et al, 2010; Milad and Quirk, 2012; Santini et al, 2008), a 
a
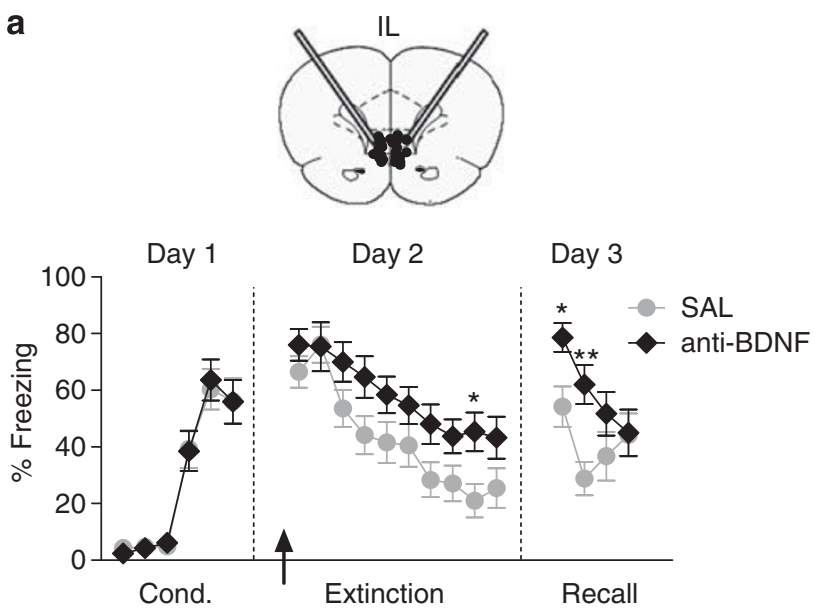

b
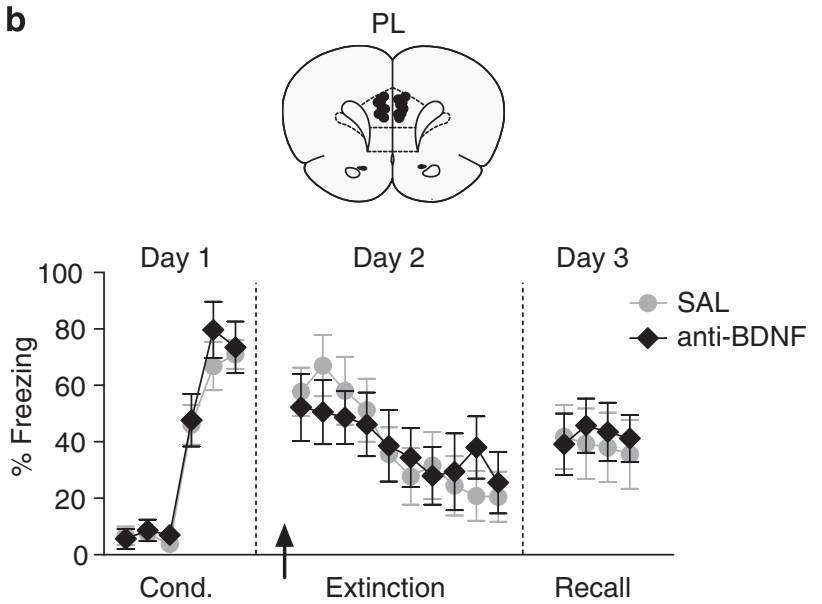

Figure 2 Brain-derived neurotrophic factor (BDNF) in infralimbic (IL), but not prelimbic (PL), is necessary for extinction. (a, upper) Coronal drawings showing the angled cannulation approach and location of the injector tips in IL. (Lower) Infusion of BDNF binding antibody (anti-BDNF) in $\mathrm{IL}$ (arrow) before extinction training impaired the acquisition of extinction and the retrieval of extinction the following day (day 3; Sal: $n=15$; anti-BDNF: $n=13$ ). (b, upper) Coronal drawings showing the location of the injector tips in PL. (Lower) Infusion of anti-BDNF in PL (arrow) before extinction training did not alter acquisition or retrieval of extinction (Sal: $n=9$; anti-BDNF: $n=9$ ). Repeated-measures analysis of variance (ANOVA) followed by Tukey's post hoc test. Data are shown as mean \pm SEM in blocks of two trials. $* P<0.05$, ${ }^{*} * P<0.0$ l.

release of BDNF into IL following vHPC infusion of BDNF would be expected to decrease fear and increase the excitability of IL neurons.

To address these questions, we infused BDNF in the vHPC of fear-conditioned rats. As shown in Figure $4 \mathrm{a}$, vHPC BDNF decreased freezing $30 \mathrm{~min}$ postinfusion $\left(\mathrm{F}_{(1,18)}=\right.$ 9.129; $P=0.007)$. In a separate group of fear-conditioned rats, we recorded IL and $\mathrm{PL}$ neuronal activity $30 \mathrm{~min}$ following vHPC infusion of BDNF. Of 16 IL neurons tested, 9 showed a significant increase in spontaneous firing rate $30 \mathrm{~min}$ following BDNF infusion, whereas 2 showed a significant decrease (paired $t$-tests $P$ 's $<0.05$; Figure $4 \mathrm{~b}$ ). In contrast, of $21 \mathrm{PL}$ neurons tested, only 1 increased, whereas 6 decreased their firing rate (paired $t$-tests $P$ 's $<0.05$; Figure 4c). The percentage of neurons showing an increase in rate was significantly greater in IL than in PL (Fisher's exact, $P<0.001$ ), but there was no significant group difference in the percentage of neurons showing reduced rate (Fisher's exact, $P=0.42$ ). These results suggest that BDNF in vHPC may enhance extinction by increasing IL activity.

\section{DISCUSSION}

In the present study, we demonstrated that BDNF in IL, but not PL, is both necessary and sufficient for extinction of older, as well as recent fear memories. We also showed that extinction training increases BDNF levels in vHPC neurons, and that BDNF infused into vHPC induces extinction and increases the firing rate of IL neurons. These findings provide key support for the hypothesis that extinction depends on the release of BDNF from hippocampal inputs to IL.

IL has been implicated in extinction of conditioned fear using pharmacological, electrophysiological, and stimulation approaches (Milad and Quirk, 2012). Furthermore, infusing BDNF into IL induces fear extinction in the absence of training (Peters et al, 2010). Exogenous BDNF has a half-life of approximately $3 \mathrm{~h}$ in the brain (Fukumitsu et al, 2006), suggesting that the observed reduction in freezing one day following infusions was not due to residual exogenous BDNF. In the adjacent PL, BDNF has been shown to be necessary for the consolidation of fear conditioning (Choi et al, 2010), and other studies have shown that PL undergoes plasticity following fear extinction (Kim et al, 2011; Parsons et al, 2010; Santini et al, 2004). Thus, our previous finding that infusion of BDNF into IL induces extinction could be due to backflow to PL. In the present study, therefore, we used an angled cannula approach which reduces backflow to PL (Sierra-Mercado et al, 2011). Although we cannot completely exclude the possibility of spread to PL by diffusion, infusing BDNF or anti-BDNF directly into PL had no effect on extinction. This agrees with previous findings that transgenic mice lacking BDNF in PL and other neocortical regions extinguish normally (Choi et al, 2010).

Molecular processes involved in consolidation of aversive learning can last hours to days (Katche et al, 2013; Riedel et al, 2000). Thus, it is possible that BDNF reduces freezing because it interferes with late consolidation of conditioning. Contrary to this idea, however, we observed that IL BDNF reduced freezing 2 weeks after conditioning, when most of these consolidation processes have terminated (Katche et al, 2013). From a clinical perspective, therefore, treatments that augment BDNF may be useful to alleviate the symptoms of older, as well as recent, traumatic memories.

Previous studies have shown that hippocampal BDNF is necessary for fear extinction (Heldt et al, 2007; Sakata et al, 2013). Our immunocytochemical results showed that extinction increased BDNF levels in vHPC and BA, but not in mPFC or central amygdala. This conflicts with a previous study showing that extinction training increased BDNF mRNA levels in the prefrontal cortex (Bredy et al, 2007). A possible explanation is that our immunocytochemical approach lacks the sensitivity to detect smaller extinctioninduced changes in protein levels. Our findings agree with a previous report showing increased BDNF in BLA $2 \mathrm{~h}$ after 


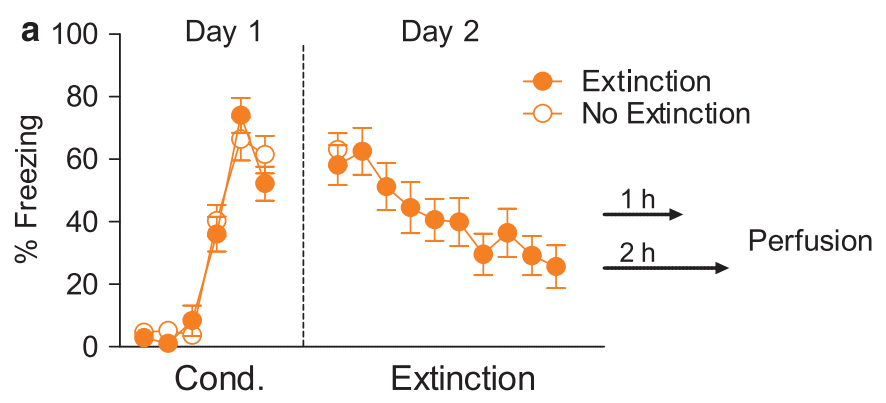

b
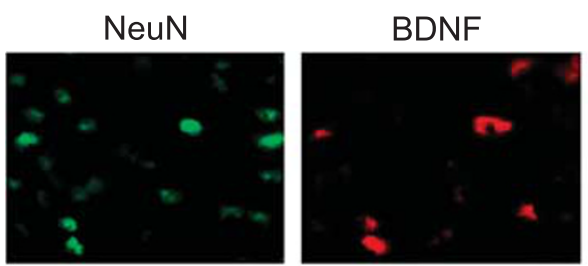

\section{Overlap}
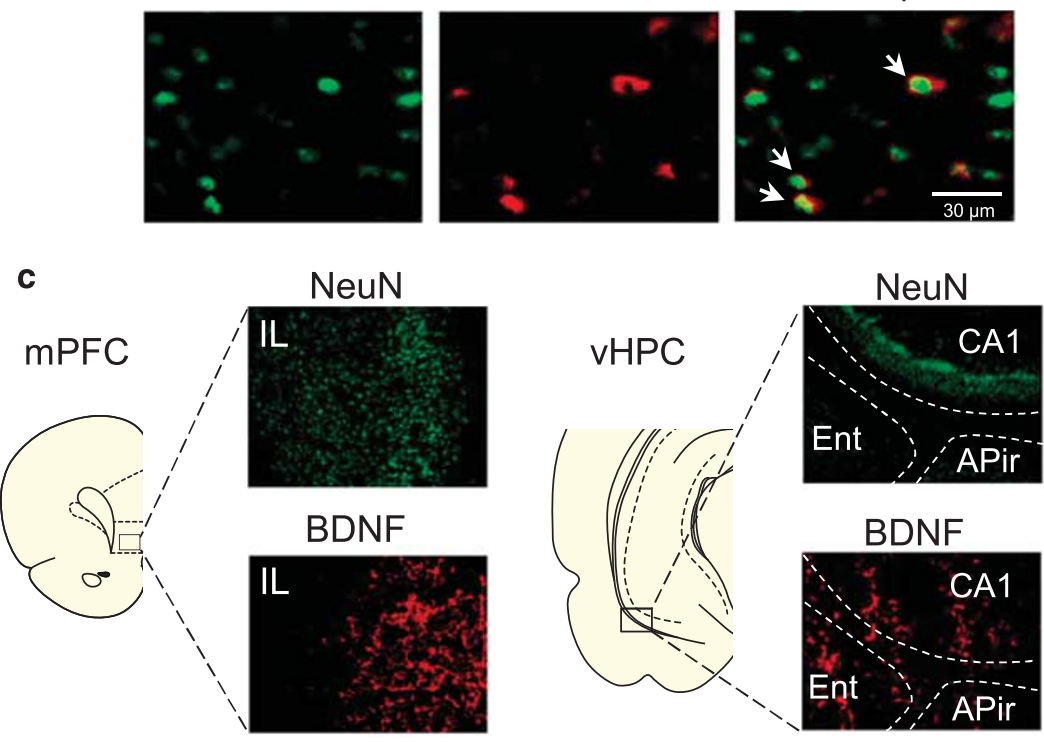

d 1 hour
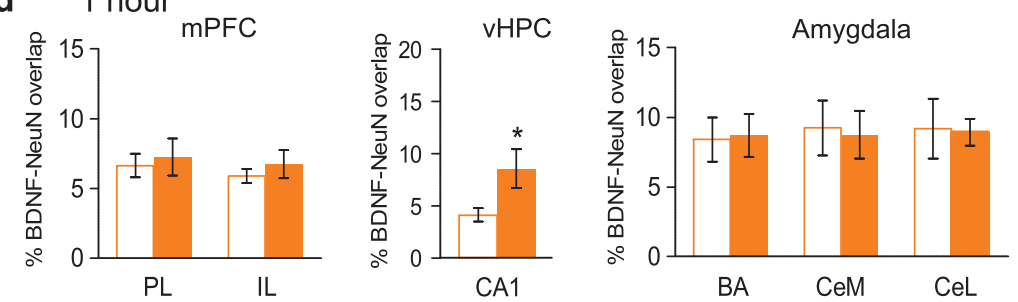

e
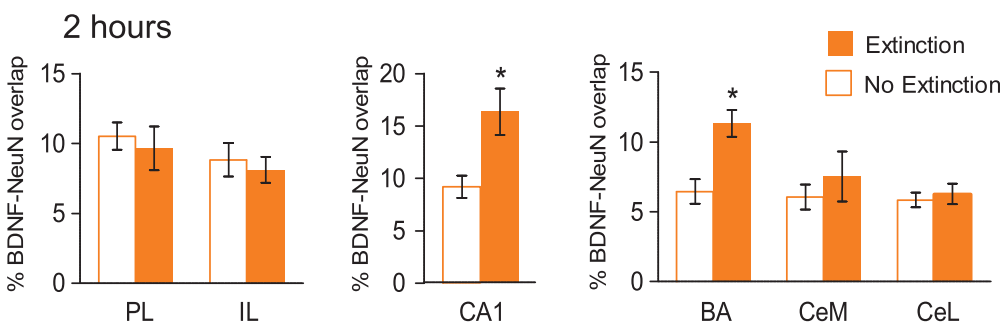

Figure 3 Extinction training increases neuronal brain-derived neurotrophic factor (BDNF) expression in the ventral hippocampus (vHPC) and basal amygdala (BA). (a) Freezing to tones during conditioning (Cond.) and extinction session (in blocks of two trials). (b) Representative micrographs showing labeling of NeuN (green), BDNF (red), and BDNF-NeuN overlap (white arrows). (c) Representative images showing labeling of NeuN and BDNF in infralimbic cortex (IL) and vHPC. ( $\mathrm{d}$ and e) Neuronal BDNF expression in the medial prefrontal cortex (mPFC), vHPC, and amygdala following exposure to 2 tones (No Extinction) or 20 tones (Extinction), as measured by percent of overlapping area between BDNF- and NeuN-labeled images. Increased neuronal BDNF was observed in the CAI subregion of the $\mathrm{vHPC}$ at both I $\mathrm{h}$ (d) and $2 \mathrm{~h}$ (e) after extinction training, compared with the No Extinction control. Increased neuronal BDNF was observed in the BA at $2 \mathrm{~h}$ after extinction training, when compared with the No Extinction group. PL, prelimbic cortex; IL, infralimbic cortex; CeM, medial portion of the central amygdala; CeL, lateral portion of the central amygdala. Two-way analysis of variance (ANOVA) between Extinction and No Extinction groups ( $\mathrm{h}: \mathrm{n}=8$ per group; $2 \mathrm{~h}: n=4$ per group). $* P<0.05$. 
a

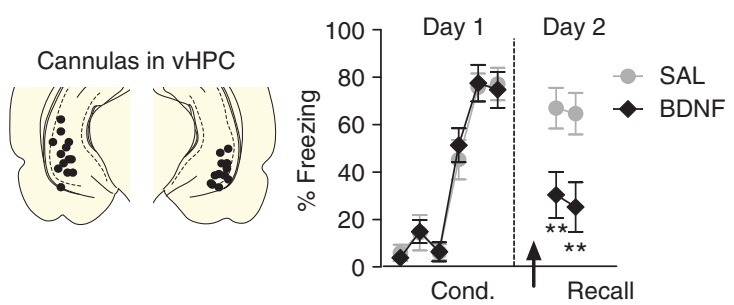

b

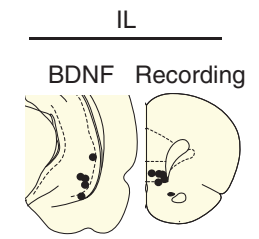

c
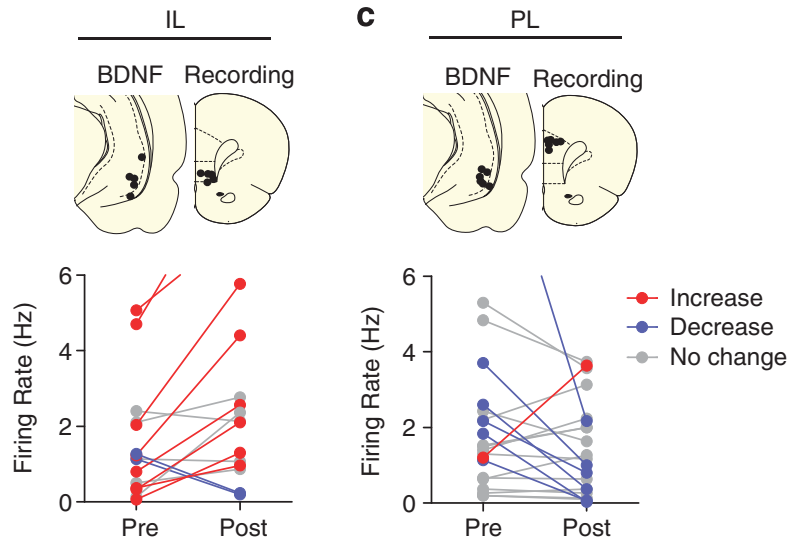

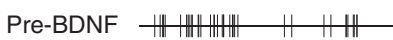

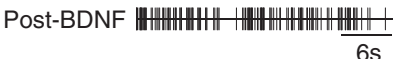

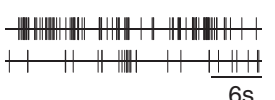

Figure 4 Brain-derived neurotrophic factor (BDNF) infused into ventral hippocampus (VHPC) reduces freezing to conditioned tones and increases the activity of infralimbic cortex (IL) neurons. (a, left) Coronal drawings showing the location of the injector tips within the VHPC. (right) Infusion of BDNF in the VHPC on day 2 (arrow) significantly decreased freezing (Sal: $n=9$; BDNF: $n=11$; repeated-measures analysis of variance (ANOVA) followed by Tukey's post hoc test; *** $<0.0$ I). (b, upper) Coronal drawings showing the location of the injector tips within the VHPC (BDNF) and the recording sites within $\mathrm{L}$ (recording). (middle) Spontaneous firing in $\mathrm{IL}$ before and $30 \mathrm{~min}$ after BDNF infusion in the $\mathrm{VHPC}$. BDNF infusion significantly increased the firing rate of eight cells (red) and decreased the rate of two cells (blue). An additional IL neuron significantly increased its firing rate from 9.33 to $17.3 \mathrm{~Hz}$ (data not shown); $n=16$. (lower) Raster plot (30s) of representative IL neuron before and after BDNF infusion in the VHPC. (c, upper) Coronal drawings showing the location of the injector tips within the VHPC (BDNF), and the recording sites within prelimbic cortex (PL) (recording). (middle) Spontaneous firing in PL before and 30 min after BDNF infusion in the VHPC. BDNF infusion significantly increased the firing rate of one PL cell (red) and decreased the rate of six cells (blue); $n=21$. (lower) Raster plot (30s) of representative PL neuron before and after BDNF infusion in the VHPC. Paired Student's $t$-test; *** $P<0.01$.

extinction (Chhatwal et al, 2006). Interestingly, another study found increased BDNF levels in the hippocampus (but not the amygdala) at an earlier timepoint (30 min) following contextual fear conditioning (Hall et al, 2000). Thus, following learning, hippocampal neurons may express BDNF initially, followed by BDNF expression in hippocampal targets such as $\mathrm{mPFC}$ and amygdala.

Increased excitability in IL following extinction has been shown to predict extinction success (Burgos-Robles et al, 2007; Chang et al, 2010; Knapska and Maren, 2009; Santini et al, 2008). We observed that infusing BDNF into vHPC increased the firing rate of IL neurons, a vHPC target. BDNF infused into the vHPC could be directly transported to IL or, more likely, could increase vHPC excitability leading to increased release of BDNF in IL. In agreement with the idea that infusion of BDNF into vHPC increases release of BDNF into IL, a previous study showed that coinfusion of antiBDNF into IL with BDNF into the hippocampus blocked BDNF induction of extinction (Peters et al, 2010). The lack of BDNF increase in IL after extinction could be due to the fact that BDNF released from vHPC terminals may not be detected by immunocytochemistry, which detects only intracellular BDNF. It is interesting that vHPC BDNF did not increase excitability in PL neurons, despite a robust projection from vHPC to PL. vHPC projections to PL could facilitate extinction independently of BDNF, through feedforward inhibition of PL (Sotres-Bayon et al, 2012).

Through its tyrosine kinase B receptor, BDNF can increase GluR1 and GluR2/3 AMPA receptor subunits (Musumeci and Minichiello, 2011; Slipczuk et al, 2009) and phosphorylate NMDA receptors (Black, 1999), both of which could enhance neuronal excitability. Increased firing of IL neurons may help drive plasticity in amygdala targets, such as the intercalated cell masses (Amano et al, 2010). Consistent with this, mice with a polymorphism (val66met) impairing BDNF release from hippocampal terminals (Chen et al, 2006) show poor extinction and impaired spike timing-dependent plasticity in IL (Pattwell et al, 2012).

The hippocampal-prefrontal pathway appears to be necessary for successful fear regulation (Hugues and Garcia, 2007; Sotres-Bayon et al, 2012) and may be deficient in post-traumatic stress disorder (PTSD; Godsil et al, 2013). Activation of the ventromedial prefrontal cortex (vmPFC), a functional homolog of the rodent IL, correlates with successful extinction (Kalisch et al, 2006; Milad et al, 2007; Phelps et al, 2004; Soliman et al, 2010). People with PTSD show decreased hippocampal volume (Bremner, 2002; Bremner et al, 1995; Chao et al, 2013; Gilbertson et al, 2002), but not if they are in remission (Chao et al, 2013). Following combat-stress, PTSD symptoms are correlated with decreased hippocampal-vmPFC connectivity (Admon et al, 2012). Thus, impaired hippocampal-vmPFC connectivity may be associated with deficient BDNF signaling, thereby exacerbating symptoms and interfering with extinction-based therapy (Felmingham et al, 2013).

\section{FUNDING AND DISCLOSURE}

This research was supported by the National Institutes of Health Grants R01-MH081975 and R37-MH058883 and the University of Puerto Rico President's Office to GJQ. The authors declare no conflict of interest.

\section{ACKNOWLEDGEMENTS}

We thank Carlos Rodríguez, Mayra Guindín-Orama, and Zarcaly Quintero for technical assistance. We also thank Jamie Peters for helpful comments on the manuscript.

\section{REFERENCES}

Admon R, Leykin D, Lubin G, Engert V, Andrews J, Pruessner J et al (2012). Stress-induced reduction in hippocampal volume and connectivity with the ventromedial prefrontal cortex are related to maladaptive responses to stressful military service. Hum Brain Mapp 34: 2808-2816. 
Alonso M, Vianna MR, Izquierdo I, Medina JH (2002). Signaling mechanisms mediating BDNF modulation of memory formation in vivo in the hippocampus. Cell Mol Neurobiol 22: 663-674.

Amano T, Unal CT, Pare D (2010). Synaptic correlates of fear extinction in the amygdala. Nat Neurosci 13: 489-494.

Andero R, Ressler KJ (2012). Fear extinction and BDNF: translating animal models of PTSD to the clinic. Genes Brain Behav 11: 503-512.

Black IB (1999). Trophic regulation of synaptic plasticity. J Neurobiol 41: 108-118.

Bredy TW, Wu H, Crego C, Zellhoefer J, Sun YE, Barad M (2007). Histone modifications around individual BDNF gene promoters in prefrontal cortex are associated with extinction of conditioned fear. Learn Mem 14: 268-276.

Bremner JD (2002). Neuroimaging studies in post-traumatic stress disorder. Curr Psychiatry Rep 4: 254-263.

Bremner JD, Randall P, Scott TM, Bronen RA, Seibyl JP, Southwick SM et al (1995). MRI-based measurement of hippocampal volume in patients with combat-related posttraumatic stress disorder. Am J Psychiatry 152: 973-981.

Burgos-Robles A, Bravo-Rivera H, Quirk GJ (2013). Prelimbic and infralimbic neurons signal distinct aspects of appetitive instrumental behavior. PLoS One 8: e57575.

Burgos-Robles A, Vidal-Gonzalez I, Santini E, Quirk GJ (2007). Consolidation of fear extinction requires NMDA receptordependent bursting in the ventromedial prefrontal cortex. Neuron 53: 871-880.

Chang CH, Berke JD, Maren S (2010). Single-unit activity in the medial prefrontal cortex during immediate and delayed extinction of fear in rats. PLoS One 5: e11971.

Chao L, Weiner M, Neylan T (2013). Regional cerebral volumes in veterans with current versus remitted posttraumatic stress disorder. Psychiatry Res 213: 193-201.

Chen ZY, Jing D, Bath KG, Ieraci A, Khan T, Siao CJ et al (2006). Genetic variant BDNF (Val66Met) polymorphism alters anxietyrelated behavior. Science 314: 140-143.

Chhatwal JP, Stanek-Rattiner L, Davis M, Ressler KJ (2006). Amygdala BDNF signaling is required for consolidation but not encoding of extinction. Nat Neurosci 9: 870-872.

Choi DC, Maguschak KA, Ye K, Jang SW, Myers KM, Ressler KJ (2010). Prelimbic cortical BDNF is required for memory of learned fear but not extinction or innate fear. Proc Natl Acad Sci USA 107: 2675-2680.

Cowansage KK, LeDoux JE, Monfils MH (2010). Brain-derived neurotrophic factor: a dynamic gatekeeper of neural plasticity. Curr Mol Pharmacol 3: 12-29.

Do-Monte FH, Rodriguez-Romaguera J, Rosas-Vidal LE, Quirk GJ (2013). Deep brain stimulation of the ventral striatum increases BDNF in the fear extinction circuit. Front Behav Neurosci 7: 102.

Felmingham KL, Dobson-Stone C, Schofield PR, Quirk GJ, Bryant RA (2013). The brain-derived neurotrophic factor Val66Met polymorphism predicts response to exposure therapy in posttraumatic stress disorder. Biol Psychiatry 73: 1059-1063.

Fukumitsu H, Ohtsuka M, Murai R, Nakamura H, Itoh K, Furukawa S (2006). Brain-derived neurotrophic factor participates in determination of neuronal laminar fate in the developing mouse cerebral cortex. J Neurosci 26: 13218-13230.

Gilbertson MW, Shenton ME, Ciszewski A, Kasai K, Lasko NB, Orr SP et al (2002). Smaller hippocampal volume predicts pathologic vulnerability to psychological trauma. Nat Neurosci 5: 1242-1247.

Godsil BP, Kiss JP, Spedding M, Jay TM (2013). The hippocampalprefrontal pathway: the weak link in psychiatric disorders? Eur Neuropsychopharmacol 23: 1165-1181.

Gunjigake KK, Goto T, Nakao K, Konoo T, Kobayashi S, Yamaguchi K (2006). Correlation between the appearance of neuropeptides in the rat trigeminal ganglion and reinnervation of the healing root socket after tooth extraction. Acta Histochem Cytochem 39: 69-77.

Hall J, Thomas KL, Everitt BJ (2000). Rapid and selective induction of BDNF expression in the hippocampus during contextual learning. Nat Neurosci 3: 533-535.

Heldt SA, Stanek L, Chhatwal JP, Ressler KJ (2007). Hippocampusspecific deletion of BDNF in adult mice impairs spatial memory and extinction of aversive memories. Mol Psychiatry 12: 656-670.

Heldt SA, Zimmermann K, Parker K, Gaval M, Ressler KJ (2014). BDNF deletion or TrkB impairment in amygdala inhibits both appetitive and aversive learning. J Neurosci 34: 2444-2450.

Herry C, Ferraguti F, Singewald N, Letzkus JJ, Ehrlich I, Luthi A (2010). Neuronal circuits of fear extinction. Eur J Neurosci 31: 599-612.

Hoover WB, Vertes RP (2007). Anatomical analysis of afferent projections to the medial prefrontal cortex in the rat. Brain Struct Funct 212: 149-179.

Hugues S, Garcia R (2007). Reorganization of learning-associated prefrontal synaptic plasticity between the recall of recent and remote fear extinction memory. Learn Mem 14: 520-524.

Kalisch R, Korenfeld E, Stephan KE, Weiskopf N, Seymour B, Dolan RJ (2006). Context-dependent human extinction memory is mediated by a ventromedial prefrontal and hippocampal network. J Neurosci 26: 9503-9511.

Katche C, Cammarota M, Medina JH (2013). Molecular signatures and mechanisms of long-lasting memory consolidation and storage. Neurobiol Learn Mem 106C: 40-47.

Kim JH, Li S, Richardson R (2011). Immunohistochemical analyses of long-term extinction of conditioned fear in adolescent rats. Cereb Cortex 21: 530-538.

Knapska E, Maren S (2009). Reciprocal patterns of c-Fos expression in the medial prefrontal cortex and amygdala after extinction and renewal of conditioned fear. Learn Mem 16: 486-493.

Milad MR, Quirk GJ (2012). Fear extinction as a model for translational neuroscience: ten years of progress. Annu Rev Psychol 63: 129-151.

Milad MR, Wright CI, Orr SP, Pitman RK, Quirk GJ, Rauch SL (2007). Recall of fear extinction in humans activates the ventromedial prefrontal cortex and hippocampus in concert. Biol Psychiatry 62: 446-454.

Mullen RJ, Buck CR, Smith AM (1992). NeuN, a neuronal specific nuclear protein in vertebrates. Development 116: 201-211.

Musumeci G, Minichiello L (2011). BDNF-TrkB signalling in fear learning: from genetics to neural networks. Rev Neurosci 22: 303-315.

Orsini CA, Kim JH, Knapska E, Maren S (2011). Hippocampal and prefrontal projections to the basal amygdala mediate contextual regulation of fear after extinction. J Neurosci 31: 17269-17277.

Ou LC, Yeh SH, Gean PW (2010). Late expression of brain-derived neurotrophic factor in the amygdala is required for persistence of fear memory. Neurobiol Learn Mem 93: 372-382.

Pape HC, Pare D (2010). Plastic synaptic networks of the amygdala for the acquisition, expression, and extinction of conditioned fear. Physiol Rev 90: 419-463.

Parsons RG, Gafford GM, Helmstetter FJ (2010). Regulation of extinction-related plasticity by opioid receptors in the ventrolateral periaqueductal gray matter. Front Behav Neurosci 4, pii 44.

Pattwell SS, Bath KG, Perez-Castro R, Lee FS, Chao MV, Ninan I (2012). The BDNF Val66Met polymorphism impairs synaptic transmission and plasticity in the infralimbic medial prefrontal cortex. J Neurosci 32: 2410-2421.

Paxinos G, Watson C (1998). The Rat Brain in Stereotaxic Coordinates. 4th edn. Academic Press: San Diego. 
Peters J, Dieppa-Perea LM, Melendez LM, Quirk GJ (2010). Induction of fear extinction with hippocampal-infralimbic BDNF. Science 328: 1288-1290.

Phelps EA, Delgado MR, Nearing KI, LeDoux JE (2004). Extinction learning in humans: role of the amygdala and vmPFC. Neuron 43: 897-905.

Quirk GJ, Mueller D (2008). Neural mechanisms of extinction learning and retrieval. Neuropsychopharmacology 33: 56-72.

Quirk GJ, Russo GK, Barron JL, Lebron K (2000). The role of ventromedial prefrontal cortex in the recovery of extinguished fear. J Neurosci 20: 6225-6231.

Riedel G, Casabona G, Platt B, Macphail EM, Nicoletti F (2000). Fear conditioning-induced time- and subregion-specific increase in expression of mGlu5 receptor protein in rat hippocampus. Neuropharmacology 39: 1943-1951.

Sakata K, Martinowich K, Woo NH, Schloesser RJ, Jimenez DV, Ji $Y$ et al (2013). Role of activity-dependent BDNF expression in hippocampal-prefrontal cortical regulation of behavioral perseverance. Proc Natl Acad Sci USA 110: 15103-15108.
Santini E, Ge H, Ren K, Pena de Ortiz S, Quirk GJ (2004). Consolidation of fear extinction requires protein synthesis in the medial prefrontal cortex. J Neurosci 24: 5704-5710.

Santini E, Quirk GJ, Porter JT (2008). Fear conditioning and extinction differentially modify the intrinsic excitability of infralimbic neurons. J Neurosci 28: 4028-4036.

Sierra-Mercado D, Padilla-Coreano N, Quirk GJ (2011). Dissociable roles of prelimbic and infralimbic cortices, ventral hippocampus, and basolateral amygdala in the expression and extinction of conditioned fear. Neuropsychopharmacology 36: 529-538.

Slipczuk L, Bekinschtein P, Katche C, Cammarota M, Izquierdo I, Medina JH (2009). BDNF activates mTOR to regulate GluR1 expression required for memory formation. PLoS One 4: e6007.

Soliman F, Glatt CE, Bath KG, Levita L, Jones RM, Pattwell SS et al (2010). A genetic variant BDNF polymorphism alters extinction learning in both mouse and human. Science 327: 863-866.

Sotres-Bayon F, Sierra-Mercado D, Pardilla-Delgado E, Quirk GJ (2012). Gating of fear in prelimbic cortex by hippocampal and amygdala inputs. Neuron 76: 804-812. 